\title{
Analysis of Magnetic Forces in Axial-Flux Permanent-Magnet Motors with Rotor Eccentricity
}

\author{
Xiaoting Zhang $(\mathbb{D}$ and Bingyi Zhang \\ School of Electrical Engineering, Shenyang University of Technology, Shenyang 110870, China \\ Correspondence should be addressed to Xiaoting Zhang; zhangxt@smail.sut.edu.cn
}

Received 27 August 2021; Revised 14 October 2021; Accepted 22 October 2021; Published 27 November 2021

Academic Editor: Sang-Bing Tsai

Copyright ( 2021 Xiaoting Zhang and Bingyi Zhang. This is an open access article distributed under the Creative Commons Attribution License, which permits unrestricted use, distribution, and reproduction in any medium, provided the original work is properly cited.

\begin{abstract}
In this study, an analytical model is established to efficiently compute the magnetic field and unbalanced magnetic pull (UMP) in axial-flux permanent-magnet motors (AFPMMs). The effects of stator slotting, end effect, and rotor eccentricity on the magnetic field and forces were investigated. Static and dynamic eccentricities are analyzed and considered in the model. An effective function of the air gap permeance was introduced for effect of the stator slots to compute the flux density. A specific coefficient function is defined to calculate the end effect. A Fourier transform is used to compute the variations of the permanent-magnet remanence and the air gap permeance due to the slotted stator opposite to a slotless stator. The unbalanced magnetic forces were evaluated as a function of the air gap magnetic field using analytical equations. The proposed analytical method dramatically reduces the model size and computational time. It can be applied to the analysis of AFPMMs and is much faster than the threedimensional finite element method (FEM). By comparing with the obtained using the FEM, the model results are validated.
\end{abstract}

\section{Introduction}

Presently, on account of the high torque density, high efficiency, and compact structure, AFPMMs have been applied in various fields and play a very important role, for instance, flywheel energy storage, wind power generation, light electrical traction, or electric vehicles, where the axial length of machine is limited and direct coupling is preferred.

The AFPMMs' modeling can be performed via analytical methods [1-7] and FEM analyses [8-10]. Because of the inherent three-dimensional (3D) structure of the AFPMMs, a full 3D finite element modeling is more suitable for calculating their performance parameters more accurately. However, computing a 3D FEM modeling generally has higher cost. Therefore, analytical modeling is often used in design and optimization. For instance, an analytical modeling of stress analysis was proposed by Shi et al. [1], and the radial force of the motor was optimized according to the modeling. Sun et al. [2] proposed an optimal control strategy for motors and proposed the derivation process of stress based on the Maxwell stress tensor method, but the modeling approach for the slotted stator adopted by the author is relatively simple. Sun et al. proposed a robust mathematical model of the relationship between skew angle of the stator slot and motor performance [3], and the performance of the motor was optimized according to this modeling; however, the influence of rotor eccentricity on the motor performance was not considered.

In AFPMMs, rotor eccentricity is likely the result of manufacturing tolerance and assembly processes that cause an eccentric air gap. The induced unbalanced magnetic forces affect machine performance dramatically if they are not well controlled. In the ideal condition with no rotor eccentricity, the length of air gap is symmetric, and the magnetic forces have uniform distribution perpendicular to the shaft axis. The axial forces are balanced and do not affect the performance of the machine significantly. On the other side, with the rotor eccentricity, the rotor become inclined and the length of air gap become asymmetric. So, the axial forces are unbalanced; meanwhile, an uneven magnetic pull that results in increased vibration, noise, and reduced bearing life is produced. As a result, rotor eccentricity plays a 
significant role in machine losses, unbalanced magnetic forces, and low NVH performance, such as noise and excess vibration. Therefore, we investigate and understand whether the influence of rotor eccentricity on the performance of AFPMMs is necessary and meaningful [4-7].

There are previous studies on UMP in radial flux machines, but UMP in axial-flux machines has not been investigated enough. Owing to the increasing need for highperformance AFPMMs, some researchers have focused on studying the forces and vibrations of machines under rotor eccentricity conditions. Bao et al. [4] developed an analytical equation to compute the flux density under impact of the rotor eccentricity. The authors used perturbation theory to compute the air gap magnetic field with the influence of eccentricity. Their analytical results were in relatively good agreement with the results of the 3D FEM modeling. However, their analytical method requires complicated computations to efficiently solve real-world problems. Zhu et al. [5] calculate the air gap flux density under static rotor eccentricity by the Fourier transform method. Their method is fairly efficient with little programming; however, it did not consider the impacts of the end effect and other rotor eccentricity conditions. Zhu et al. analyzed the magnetic forces of concentrated-winding permanent-magnet motors under eccentricity conditions and achieved the order of the eccentricity-induced force waveform using the Fourier transform [6]. Their findings provide a useful method for monitoring motor eccentricity. Araki et al. presented an analytical method for obtaining the overall air gap flux density distribution of AFPMMs [7]. First, the air gap flux density distribution of each loop is calculated and then the results of all loops are summed. Their method is simple. However, it becomes more computationally intense if the number of loops increases. Marignetti et al. proposed an analytical approach that allows for the effects of simple rotor eccentricity on the performance of AFPMMs [8], which is a quick evaluation of the generator performance suitable for online identification and have reasonable accuracy. However, the impacts of stator slots and end effects have not been studied.

In studies by Mirimani and Vansompel et al. [8-10], finite element modeling was used to analyze the performance of AFPMMs. Mirimani et al. used 3D FEM to analyze the impact of the inclined eccentricity on the magnetic forces [8]. Vansompel et al. used 3D FEM modeling to study the back electromotive force, and the impact of static eccentricity on the air gap flux density of an AFPMM [9]. The 3D FEM can accurately compute the flux-density distribution of AFPMMs under the impact of eccentricity. However, it is time-consuming and less efficient than analytical methods with similar accuracy. Vansompel et al. presented a method for computing the flux density of AFPMMs under the impact of static eccentricity by employing an equivalent remanence approach, where the degree of nonuniformity of the remanence was used to approximate the air gap flux density [10].

Based on the above literature review, precise and efficient calculation results of the distribution of magnetic flux density are necessary for magnetic force modeling, especially in the air gap region of AFPMMs. Therefore, it is necessary to develop a valid analytical method that can quickly compute the unbalanced forces of AFPMMs under rotor eccentricity conditions with high accuracy. In this study, we innovatively develop the magnetic field of the disc motor, analyse the force on the eccentricity state, which provides a reference for the stress and fatigue life analysis of bearing, mandrels, and other stress elements, and develop an analytical method that can more quickly compute the performance of AFPMMs under the impact of eccentricity with the required accuracy.

In this study, we established an analytical model to obtain the air gap magnetic field of an AFPMM with the rated power of $10 \mathrm{~kW}$ and the rated rotational speed of $1500 \mathrm{r} / \mathrm{min}$ under ideal conditions. A specific magnetic permeance function was first developed to calculate the air gap performance considering the effects of the slotted stator and the end effect of the machine. The change in the air gap length under rotor eccentricity was also analyzed. Compute the air gap permeance by the Fourier transform method. Then, an analytical model was established to obtain the air gap magnetic field under eccentricity conditions. Finally, the analytical modeling is used to compute the unbalanced magnetic forces due to rotor eccentricities, considering both the end effect and slotted stators.

\section{Model of an AFPMM}

Figure 1 shows a 3D model of the AFPMM. Table 1 listed key parameters. The machine has a stator rotor-stator structure with dual stators and a single rotor. The permanent magnet $(\mathrm{PM})$ is fan shaped. The stator has a fractional-slot concentrated winding.

\section{Model for Computing Air Gap Magnetic Field}

To analytically compute the magnetic forces precisely, the first process about modeling an AFPMM is computing the air gap magnetic flux density. All other performance parameters of the motor can be derived from it, for example, the induced winding voltages, torque, and magnetic forces. Therefore, the accuracy of the magnetic flux density analysis affected the accuracy of the magnetic force modeling directly.

3.1. The Air Gap Magnetic Field under Ideal Condition with No Eccentricity. To simplify the analytical model of the AFPMM, we set the following assumptions:

(1) The saturation effect and reluctance of the stator iron core is neglected

(2) The eddy current effect is neglected

(3) The demagnetization curve of the permanent magnet is linear

The air gap flux density of the AFPMM under the impacts of slotting and the end effect considered in no loading is expressed as follows: 


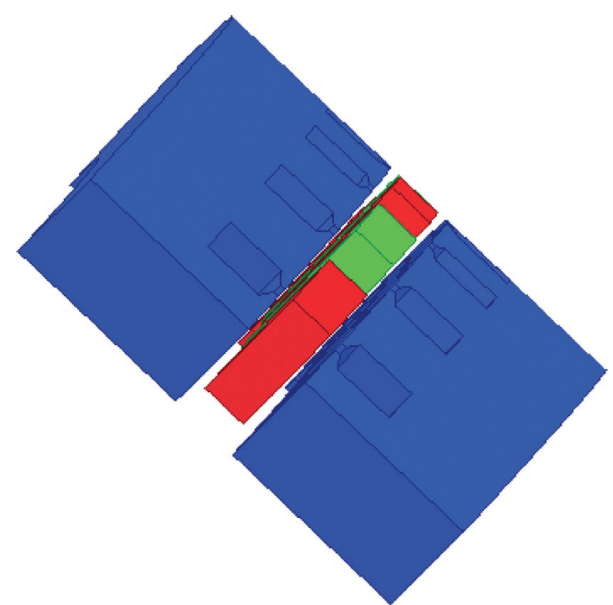

FIGURE 1: The three-dimensional geometry prototype of the studied AFPMM.

TABLe 1: Parameters of AFPMM.

\begin{tabular}{lc}
\hline Parameters & Value \\
\hline Rated power $(\mathrm{kW})$ & 10 \\
Number of pole pairs & 10 \\
Length of air gap (mm) & 1 \\
Rated rotational speed (r/min) & 1500 \\
Number of slots & 24 \\
Pole-arc to pole-pitch ratio & 0.83 \\
Machine structure & Dual stators' single rotor \\
\hline
\end{tabular}

$$
B_{g}(r, \theta)=B_{\mathrm{sg}}(r, \theta) \lambda_{g}(r, \theta) \xi(r),
$$

where $B_{g}(r, \theta)$ is air gap flux density, $B_{\text {sg }}(r, \theta)$ is the ideal flux density function with slotless stators and no end effect, $\lambda_{g}(r, \theta)$ is the function of the air gap permeance with slotted stators, and $\xi(r)$ is the coefficient function that represents the end effect contribution to the flux density.

Figure 2 shows the analysis of a given radius of the motor in a two-dimensional (2D) plane, which is a cross-section modeling in order to compute the distribution of air gap flux density of a slotless-stator AFPMM. In this case, the dual stator had smooth surfaces with no slots.

Figure 3 shows the remanence distribution of the permanent magnet based on the $2 \mathrm{D}$ model. As shown in Figure 3 , the remanence is equal to zero between two adjacent poles.

Fourier decomposition of the remanence function shown in Figure 3 is obtained as follows:

$$
M(x)=\sum_{n}^{\infty} \frac{4 B_{r}}{n \pi \mu_{0}} \sin \frac{n \pi \alpha_{p}}{2} \cos \frac{n \pi x}{\tau},
$$

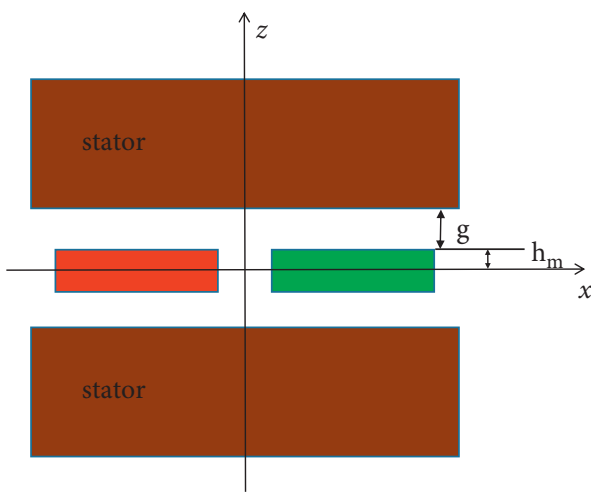

FIgURE 2: The two-dimensional cross-section model of an AFPMM with two slotless stators.

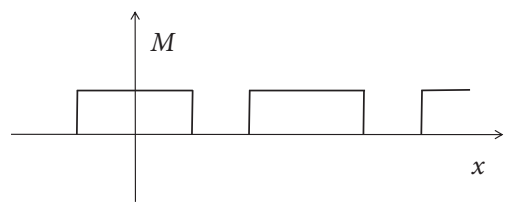

FIGURE 3: PM remanence distribution between two adjacent poles.

where $\mu_{0}=4 \pi \times 10^{-7}(\mathrm{H} / \mathrm{m})$ is vacuum permeability, $B_{r}$ is the residual flux density of the permanent magnet, $\alpha_{p}$ is the pole-arc coefficient, $\tau$ is the pole pitch, and $n$ is the number of air gap flux density hormonics. By calculating the curl of the remanence, the current density $J_{M}$ can be expressed as follows:

$$
J_{M}=\nabla \times M(x) .
$$

In the 2D AFPMM model shown in Figure 2, the magnetic vector potentials in the air gap and permanentmagnet areas satisfy the Laplace and Poisson equations as follows:

$$
\begin{aligned}
& \frac{\partial^{2} A_{1}}{\partial x^{2}}+\frac{\partial^{2} A_{1}}{\partial y^{2}}=0, \\
& \frac{\partial^{2} A_{2}}{\partial x^{2}}+\frac{\partial^{2} A_{2}}{\partial y^{2}}=-\mu_{M} J_{M} .
\end{aligned}
$$

Based on equations (4) and (5), the magnetic vector potentials of their derivatives can be solved. The ideal flux density can be expressed as

$$
B_{\text {sg }}=\sum_{n}^{\infty} \frac{-8 B_{r} \sin \left(n \pi \alpha_{p} / 2\right) \sinh \left(n \pi h_{m} / \tau\right) \cos (n \pi x / \tau)}{n \pi\left(\mu_{r}+1\right) \sinh \left[n \pi\left(h_{m}+g\right) / \tau\right]+n \pi\left(\mu_{\mathrm{r}}-1\right) \sinh \left[n \pi\left(g-h_{m}\right) / \tau\right]} \vec{z}
$$


where $\mu_{r}$ is the relative permeability of the permanent magnet, $g$ is the length of air gap, $h_{m}$ is the thickness of permanent magnet, and $g$ and $h_{m}$ are shown in Figure 2.

To simplify the calculation, under the ideal conditions with no eccentricity, the flux density $B_{\mathrm{sg}}$ in equation (6) can be transformed in the cylindrical coordinate system and expressed in equation (7), based on its cylindrical geometric characteristics of the AFPMM, and $p$ is the number of pole pairs:

$$
B_{\mathrm{sg}}=\sum_{n}^{\infty} \frac{-8 B_{r} \sin \left(n \pi \alpha_{p} / 2\right) \sinh \left(n p \pi h_{m} / \tau\right) \cos (n p \theta)}{n \pi\left(\mu_{r}+1\right) \sinh \left[n \pi \mathrm{p}\left(h_{m}+g\right) / \tau\right]+n \pi\left(\mu_{\mathrm{r}}-1\right) \sinh \left[n p \pi\left(g-h_{m}\right) / \tau\right]} \vec{z}
$$

3.2. Effect of Stator Slots on Air Gap Permeance. Stator slotting causes variations in the magnetic field have different modes. One is decreasing the average magnetic flux of each pole. The other is slotting affects the distribution of flux in the air gap. In conclusion, slotting leads to a nonhomogeneous distributed air gap flux density. At positions opposite to a slot opening with low permeance, the flux density have a smaller value than which is at opposite positions to the tooth tips with high permeance. The slotting effect results in (1) an increase in the reluctance in the slotted areas, (2) a change in the direction of the magnetic field, (3) a reduction in the airgap flux density, and (4) an increase in the harmonics of the air-gap flux density owing to the effect of the tooth harmonics. To calculate the actual air gap flux density with the slotting effect, we define the air gap permeance as follows:

$$
\lambda(r, \theta)=\left\{\begin{array}{l}
1 \\
\frac{g+h_{m} / \mu_{r}}{\pi r\left|\theta-\theta_{1}\right| / 2+g+h_{m} / \mu_{r}},
\end{array}\right.
$$

where $\theta_{1}$ is the distance from the tooth edge to the tooth center.

Fourier decomposition is then performed for equation (8), and the air gap permeance $\lambda_{\mathrm{ag}}$ can be computed as follows:

$$
\lambda_{\mathrm{ag}}=\lambda_{0}+\sum_{n=1}^{\infty} \lambda_{n} \cos \left[n Q_{1}\left(\theta+\phi_{0}\right)\right]
$$

where $Q_{1}$ is the number of stator slots, $\lambda_{0}$ is the directcurrent component of the air gap permeance, and $\varphi_{0}$ is the initial phase.

Figure 4 shows the calculated result of the air gap permeance waveform obtained by equation (9).

\subsection{Impact of the End Effect on Air Gap Magnetic Flux Density} with Slotted Stator. Figure 5 reveals the calculated air gap flux density waveform at the central line of the AFPMM magnetic poles using finite element modeling (FEM). As shown in the figure, the flux density values at the ends are lower than those at other locations, which indicate the significance of the end effect. So, the influence of the end effect is very important for the flux density and cannot be neglected in analysis.

To consider the effects of both slotted stators and the end effect, based on equations (1) and (7), we can calculate the air gap flux density in the AFPMM under the no-load condition using the following equation:

$$
\xi(r)=\frac{\sinh \left(R_{o}-R_{i} / \alpha\right)}{\cosh \left(R_{o}-R_{i} / \alpha\right)+\cosh \left(R_{o}+R_{i}-2 r / \alpha\right)},
$$

where $R_{o}$ is the outer radius of stator and $R_{i}$ is the inter radius of stator. After solving equations (9)-(10), the equivalent air gap flux density distribution was calculated. Figure 6 shows a comparison of the air gap flux density obtained using the analytical method and FEM which considered the influence of end effect and stator slotting. As a result, air gap flux density distribution obtained by the two methods has the same trend, the agreement was very good, and the maximum deviation rate of the two methods was $1.33 \%$; it verified the correctness and validity of the analytical method.

\section{Analysis of the AFPMM Eccentricity}

4.1. Analysis of Magnetic Field and Forces under Eccentricity Condition. The AFPMM may have three different eccentricities: static, dynamic, and mixed eccentricities. Figure 7 show the relationship between rotating axis and physical axis with static eccentricity and dynamic eccentricity. For an AFPMM with static eccentricity, the geometric center of the stator does not coincide with that of the rotor, but the rotor rotates around its geometric center axis. For an AFPMM with dynamic eccentricity, the geometric center of the stator does not coincide with that of the rotor, and the rotor rotates around the axis of the stator. Mixed eccentricity refers to the presence of both static and dynamic eccentricities. Three different eccentricities influenced the length of air gap.

Eccentricities lead to nonuniformity of the air gap in the AFPMMs, and it will result in a change of the magnetic permeance, flux density, and other parameters in the air gap between the rotor and stator. This causes UMP and uneven stress distribution. The distortion of the forces around the rotor produces undesirable vibrations and noise in the AFPMM.

The directional angle of the rotor eccentricity is defined in Figure 8, where $O_{0}$ is the geometric center of the stator, $O_{r}$ is the geometric center of the rotor, and $\alpha$ is the directional angle of the rotor eccentricity.

In static eccentricity, based on its characteristics, the rotor rotates around its shaft. The length of the air gap was independent of rotor rotation. It depends on the rotor 


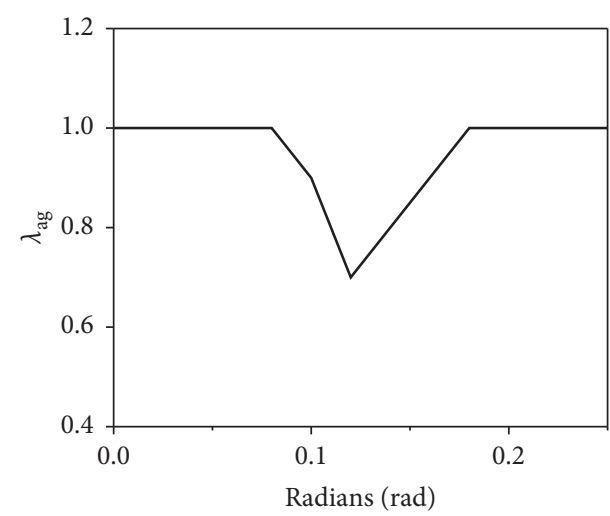

Figure 4: Air gap permeance waveform.

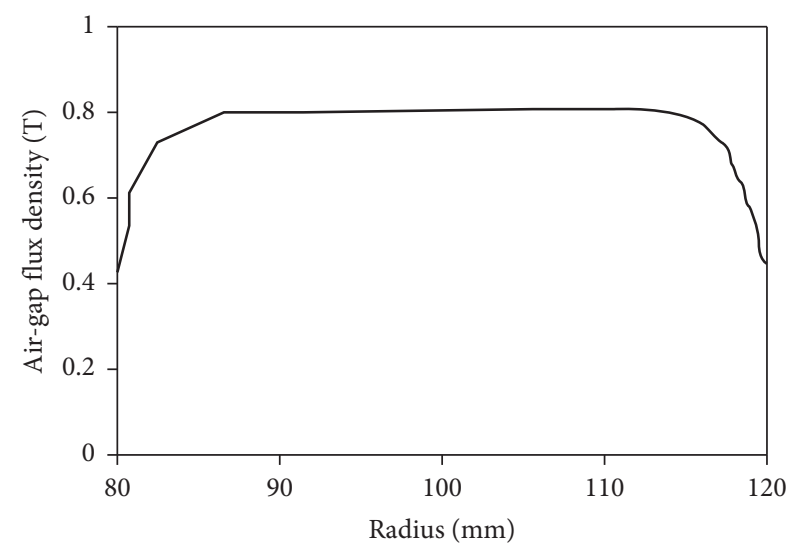

FIGURE 5: Air gap magnetic density waveform obtained using the FEM.

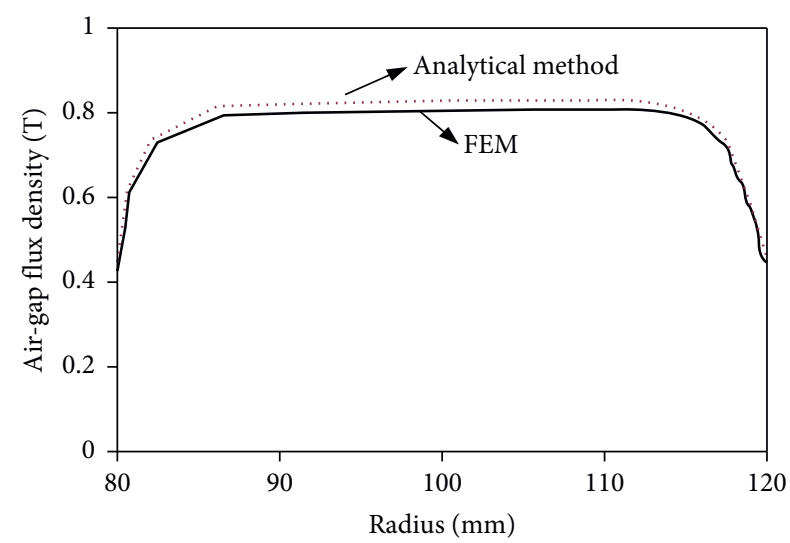

Figure 6: Comparison of flux density results.

angular position angle $\theta$ and the eccentricity directional angle $\alpha$. The length of air gap is defined as follows:

$$
g_{s}=g-e \cos (\theta-\alpha)=g[1-\varepsilon \cos (\theta-\alpha)],
$$

where $e$ is the maximum eccentric distance between the stator and the rotor and $\varepsilon$ is expressed as coefficient of the eccentricity, which is defined as $\varepsilon=e / g$.

Using the calculated air-gap length, the magnetic permeance can be obtained by

$$
\lambda_{\mathrm{s}}=\frac{1}{g[1-\varepsilon \cos (\theta-\alpha)]} .
$$

The permeance function, $\lambda_{s}$ can be expanded as a Fourier series:

$$
\lambda_{s}=\lambda_{0}+\sum_{n}^{\infty} \lambda_{1} \cos [n(\theta-\alpha)] .
$$

Figure 9 shows the air-gap flux density waveforms in the case of static eccentricity calculated using our analytical method and FEM simulation. They are in very good agreement, which validates the accuracy of the proposed analytical model.

In dynamic eccentricity, the rotor rotates around the axis of the stator bore. The length of the air gap is affected not only by the rotor angular position and its initial eccentricity angle but also by the rotor rotation cycling. It can be calculated by

$$
g_{d}=g-e \cos (\theta-\alpha-\omega t)=g[1-\varepsilon \cos (\theta-\alpha-\omega t)],
$$

where $\omega$ is the rotor rotation angular speed and $t$ is the rotation time.

Based on the calculated air-gap length $g_{d}$, the magnetic permeance can be obtained by

$$
\lambda_{d}=\frac{1}{g[1-\varepsilon \cos (\theta-\alpha-\omega t)]} .
$$

The permeance function, $\lambda_{d}$, can be transformed as a Fourier series:

$$
\lambda_{d}=\lambda_{0}+\sum_{n}^{\infty} \lambda_{n} \cos n(\theta-\alpha-\omega t) .
$$

Figure 10 shows the air-gap flux density waveforms under the condition of dynamic eccentricity calculated using our proposed analytical method and FEM simulation. This agreement was very good.

4.2. Magnetic Force Analysis. In the ideal condition with no rotor eccentricity, the length of air gap is a constant value around the shaft axis. The air gap flux was evenly distributed. The magnetic forces between the rotor and stator are uniformly distributed. Under the influence of eccentricity condition, the axial forces in the stator and rotor become uneven, resulting in an UMP. In particular, the axial force increases in small air gap areas and decreases in large air gap areas. The axial force of the AFPMM can be calculated by

$$
P_{z}=\frac{B_{z}^{2}-B_{\theta}^{2}}{2 \mu_{0}} \text {. }
$$

The magnetic force on a small element in the rotor can be obtained as

$$
\mathrm{d} F_{z}=P_{z} \mathrm{~d} S=P_{\mathrm{z}} r \mathrm{~d} r \mathrm{~d} \theta .
$$

The UMP in the $x$-and $y$-directions can be computed by integrating the resulting force $\mathrm{d} F_{z}$ as follows: 


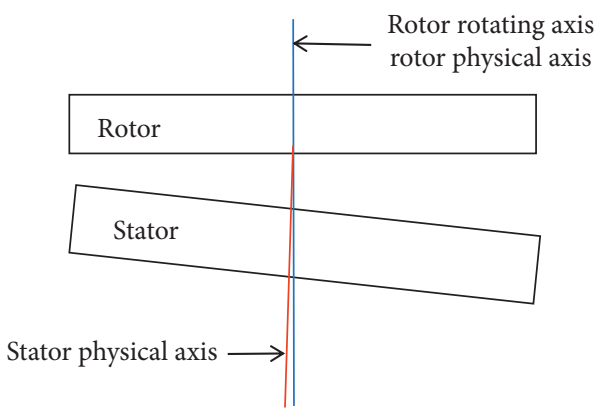

(a)

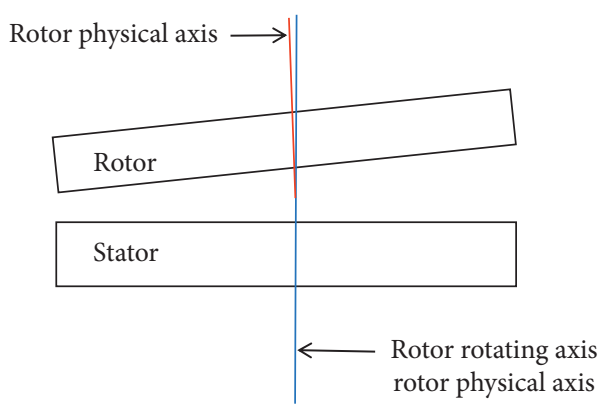

(b)

FIgUre 7: Two types of rotor eccentricities adopted. (a) Static eccentricity. (b) Dynamic eccentricity.

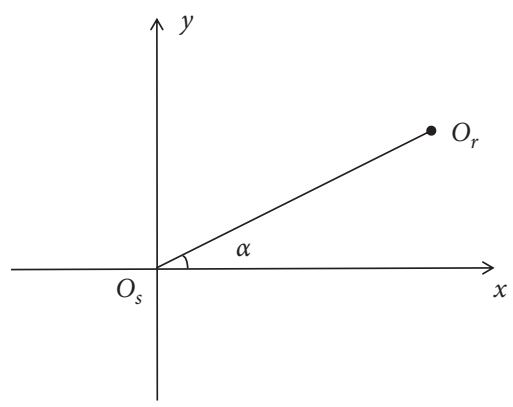

Figure 8: Schematic of the directional angle $\alpha$ of the rotor eccentricity.

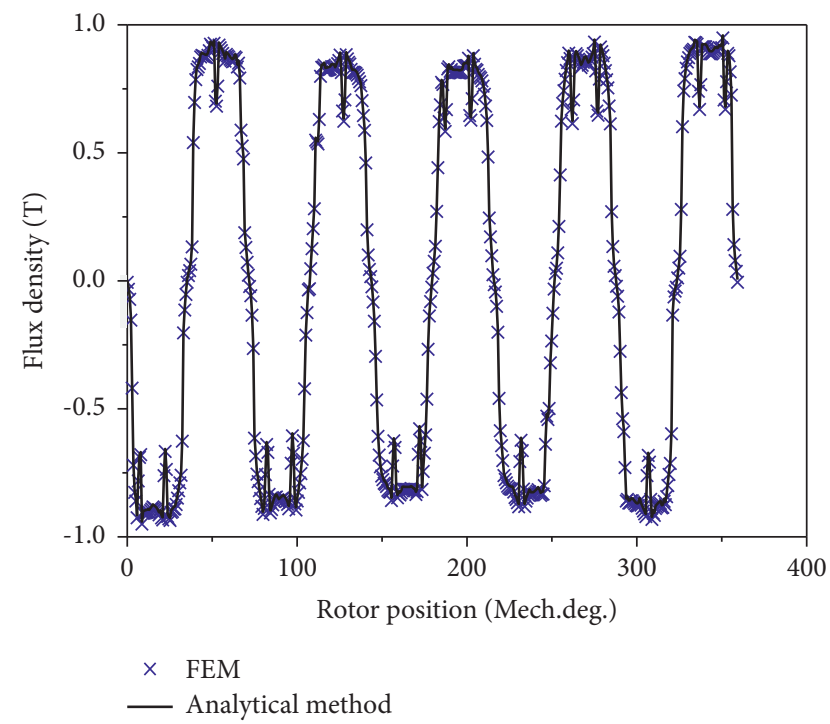

FIgURE 9: Comparison of calculated air-gap flux density under the impact of a static eccentricity using the proposed analytical method and FEM simulation.

$$
\begin{aligned}
& F_{x}=\int_{0}^{2 \pi} \int_{r_{i}}^{r_{o}} P_{z} r \sin \theta \mathrm{d} r \mathrm{~d} \theta \\
& F_{y}=\int_{0}^{2 \pi} \int_{r_{i}}^{r_{o}} P_{z} r \cos \theta \mathrm{d} r \mathrm{~d} \theta .
\end{aligned}
$$

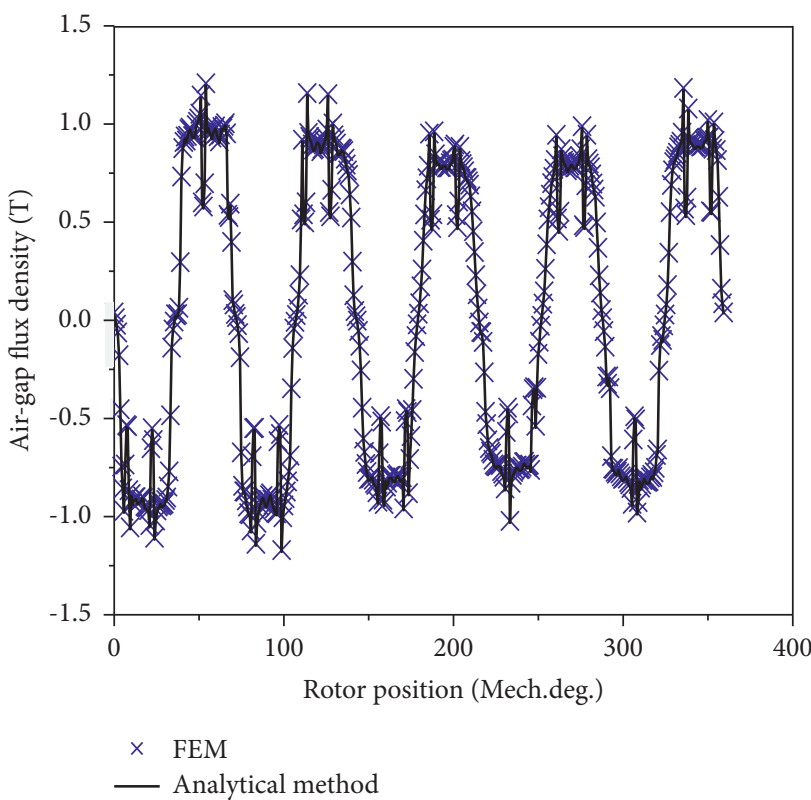

FIGURE 10: Comparison of calculated air-gap flux density under the impact of a dynamic eccentricity using the proposed analytical method and FEM simulation.

Figure 11 shows the result of UMP with influence of static eccentricity. The static eccentricity is assumed in the $x$ direction only, so an UMP occurs only in the $x$-direction. The results show that, as the coefficient of eccentricity increases, the UMP gradually increases. In addition, the UMP did not change with time. The agreement between the analytical method and FEM simulation was very good.

The percentage value is the eccentricity coefficient $\varepsilon$, defined in equation (11). When the eccentricity is set at $100 \%$, the rotor deviation length is half that of the pole boot.

Figures 12 and 13 show the influence of dynamic eccentricity on the computed unbalanced magnetic pull components in the $x$-direction and y-direction, $F_{x}$ and $F_{y}$, respectively. Unlike static eccentricity, under the impact of dynamic eccentricity, the UMP occurs in both the $x$-and $y$ directions and varies periodically over time. It has similar case to static eccentricity; as dynamic eccentricity increases, the UMP gradually increases. 


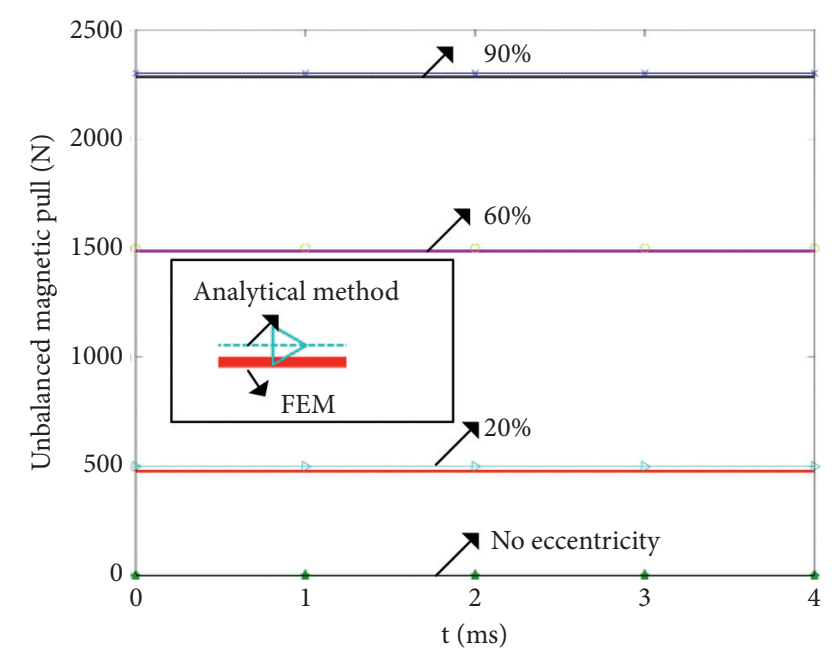

FIgURE 11: Distribution of UMP along the horizontal axis under the impact of a static eccentricity.

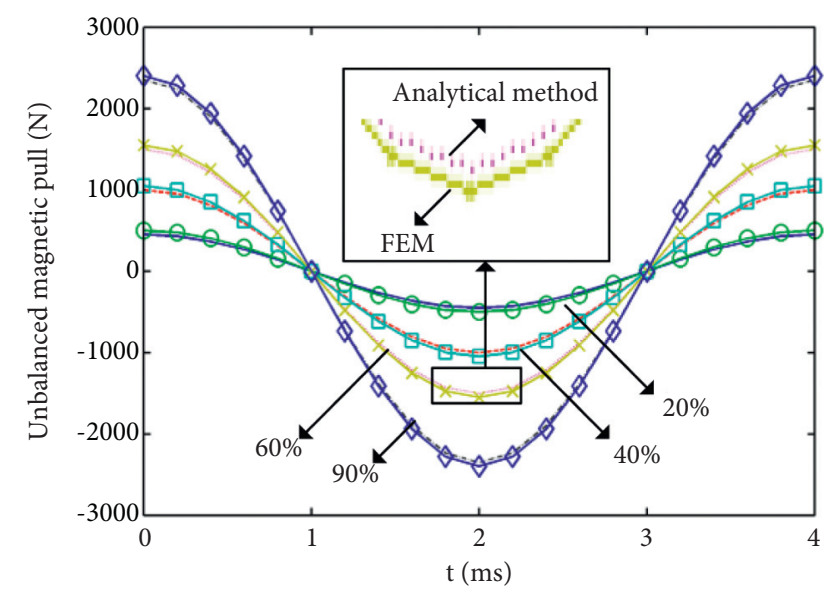

FIGURE 12: Distribution of UMP along the horizontal axis under the impact of a dynamic eccentricity.

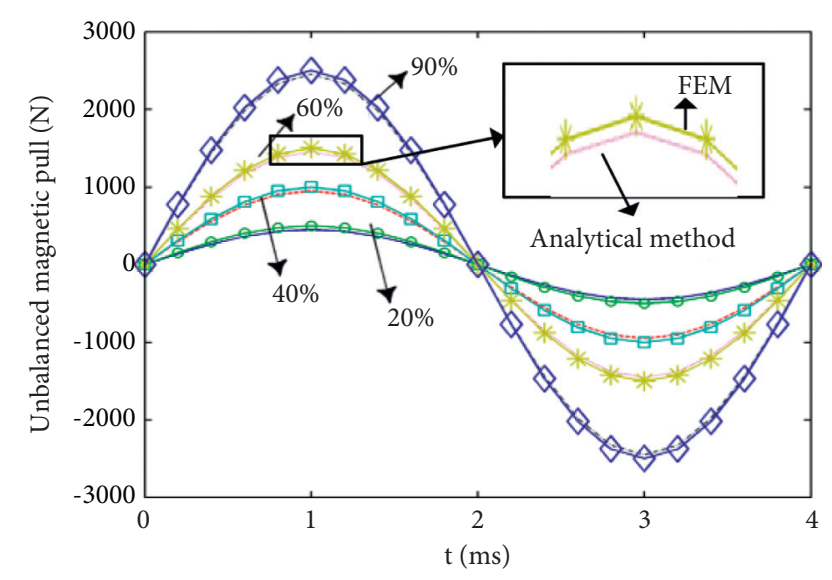

Figure 13: UMP in the $y$-direction.

In this study, an efficient analytical computational model to calculate the air-gap magnetic field and forces of AFPMMs is discussed. The effects of the stator slotting and static and dynamic eccentricity and end effect on the variations of the air-gap permeance, flux density, and magnetic forces were fully investigated and adopted in the model. The effective functions are induced to compute the variations in the PM remanence and air gap. The analytical model results were validated by comparison with the results from FEM calculations. We found that the unbalanced magnetic pull increased as the eccentricity coefficient increased. Under the impact of the static eccentricity condition, the UMP does not change with time, whereas under the impact of a dynamic eccentricity condition, the UMP varies periodically over time. Compared to the time-consuming 3D FEM calculation, our proposed analytical method can be applied to various AFPMMs for the calculation of the air-gap magnetic field and forces with high efficiency and accuracy.

Stator grooving leads to an increase in local reluctance, which leads to an increase in the air gap magneto-dense harmonics. In this study, the correction coefficient is introduced to correct the influence of the end effect of the axial-flux permanent-magnet motor. The accuracy of the correction coefficient is verified by comparison with the finite element calculation results.

\section{Conclusion}

We discussed an efficient analytical computational model to calculate the air-gap magnetic field and forces of AFPMMs in the study. The model incorporated functions for computing the air-gap permeability and the end effect to account for the impact of slotting and the end effect on the air-gap flux density. Based on this, the relationship between the air gap length and the eccentricity was established, the air-gap flux density under the impact of eccentricity was computed, and the stress in the machine under the impact of eccentricity was analyzed. The results can be summarized as follows:

(1) An analytical model for computing the air-gap magnetic field of the AFPMM was developed by performing Fourier decomposition on the functions that express the air-gap permeability and the end effect and using Fourier functions in the analytical computations. Compared with the use of the timeconsuming 3D FEM to compute the air-gap magnetic field of the AFPMM, the analytical method can compute the air-gap magnetic field of the AFPMM more accurately and quickly.

(2) The presence of an eccentricity in the AFPMM leads to a distortion in the air-gap magnetic field and inequality between the magnetic fields of any two pole pairs. A dynamic eccentricity leads to a distortion in the air-gap magnetic field larger than that of a static eccentricity.

(3) An eccentricity in the AFPMM produces an UMP. As the eccentricity increases, the UMP increases. Under the impact of a static eccentricity, the UMP does not vary with time, whereas under the impact of a dynamic eccentricity, the UMP varies periodically with time. 
(4) Stator grooving leads to the increase of local reluctance, which leads to the increase of air gap magneto-dense harmonics. In this paper, the correction coefficient is introduced to correct the influence of the end effect of the axial-flux permanentmagnet motor. By comparing with the finite element calculation results, the accuracy of the correction coefficient is verified.

\section{Data Availability}

No data were used to support this study.

\section{Conflicts of Interest}

The authors declare that they have no conflicts of interest in this study.

\section{Acknowledgments}

This research was funded by the National Key Scientific Instrument and Equipment Development Projects, China (Grant no. 2011YQ030114).

\section{References}

[1] Z. Shi, X. D. Sun, G. Lei, Z. B. Yang, Y. G. Guo, and J. G. Zhu, "Analysis and optimization of radial force of permanent magnet synchronous hub motors," IEEE Transactions on Magnetics, vol. 56, no. 2, 2020.

[2] X. Sun, Z. Jin, Y. Cai, Z. Yang, and L. Chen, "Grey Wolf optimization algorithm based state feedback control for a bearingless permanent magnet synchronous machine," IEEE Transactions on Power Electronics, vol. 35, no. 12, pp. 1363113640, 2020.

[3] X. Sun, Z. Shi, G. Lei, Y. Guo, and J. Zhu, "Multi-objective design optimization of an IPMSM based on multilevel strategy," IEEE Transactions on Industrial Electronics, vol. 68, no. $1,2020$.

[4] X. H. Bao, Q. Lu, and H. Wang, "Study on air-gap eccentricity fault in large submersible motors with tooth magnetic field analysis," Transactions of China Electrotechnical Society, vol. 31, no. 8, pp. 90-95, 2016.

[5] Z. Q. Zhu, L. J. Wu, and M. Jamil, "Influence of pole and slot number combinations on cogging torque in permanentmagnet machines with static and rotating eccentricities," in Proceedings of the Energy Conversion Congress and Exposition (ECCE), 2013, IEEE, Denver, CO, USA, September 2013.

[6] Z. Q. Zhu, L. J. Wu, and M. L. Mohd Jamil, "Influence of Pole and slot number combinations on cogging torque in permanent-magnet machines with static and rotating eccentricities," IEEE Transactions on Industry Applications, vol. 50, no. 5, pp. 3265-3277, 2014.

[7] S. Araki, T. Kondou, and A. Yamagiwa, "Analyses of electromagnetic forces of concentrated winding permanent magnet brushless motors with rotor eccentricits," IEEE International Conference on Electrical Machines and Systems, vol. 10, no. 2, pp. 1-4, 2009.

[8] S. M. Mirimani, A. Vahedi, and F. Marignett, "Effect of inclined static eccentricity fault in single stator-single rotor axial flux permanent magnet machines," IEEE Transactions on Magnetics, vol. 48, no. 1, pp. 144-149, 2012.
[9] H. Vansompel, P. Sergeant, and L. Dupre, "A multilayer 2-D2-D coupled model for eddy current calculation in the rotor of an axial-flux PM machine," IEEE Transactions on Energy Conversion, vol. 27, no. 3, pp. 784-791, 2012.

[10] H. Vansompel, P. Sergeant, and L. Dupie, "Effect of segmentation on eddy-current loss in permanent-magnets of axial-flux PM machines using a multilayer-2D: 2D coupled model," in Proceedings of the 20th International Conference on Electrical Machines, Marseille, France, September 2012. 